\title{
The effect of stimulus probability on the speed and accuracy of naming alphanumeric stimuli
}

\author{
KEITH E. STANOVICH and ROBERT G. PACHELLA \\ Human Performance Center, University of Michigan, 330 Packard Road, Ann Arbor, Michigan 48104
}

\begin{abstract}
Previous studies that have investigated the effect of stimulus probability on the time to name alphanumeric stimuli have yielded inconsistent results. Experiments purporting to show the independence of naming reaction time from stimulus probability have displayed error rates that were correlated with probability levels. In order to investigate the possible influence of the speed-accuracy trade-off in these results, the effect of stimulus probability on a naming task was studied under different instructional conditions. When accuracy was emphasized, a significant effect of stimulus probability on reaction times was obtained. When speed was emphasized by the use of a response-time deadline procedure, a significant effect of stimulus probability on error rates was observed. It was concluded that stimulus probability does affect the time to name alphanumeric stimuli, that the effect can manifest itself in both reaction times or error rates, and that models predicting naming time to be independent of stimulus probability must be modified, possibly by elaborating the processing operations of the stimulus identification stage.
\end{abstract}

One of the most complete and explicit models of the component mental operations involved in information processing tasks is that developed by Theios (1975). The model postulates that an initial stimulus input stage is followed by an identification stage which yields the name code of the stimulus. The name code is obtained via a content-addressable memory retrieval system which operates independently of variables associated with the informational content of the display, such as stimulus probability and number of stimulus alternatives. Identification time can be affected, however, by the physical properties of the stimulus (e.g., clarity or contrast).

The sequence of processing subsequent to stimulus identification is dependent upon the type of response required of the subject. In tasks with highly compatible $S-R$ codes, such as naming verbal materials, the name code is assumed to be located in a memory location near the response code. The name code to response code translation is accomplished via a content-addressable retrieval process from longterm memory. In the case of highly compatible naming tasks, this retrieval process takes a relatively constant amount of time and is independent of stimulus probability and number of stimulus alternatives.

In less compatible tasks, such as executing manual responses (e.g., pressing buttons) to alphanumeric stimuli, a search through long-term memory may be

This research was supported by the Advanced Research Projects Agency, U.S. Department of Defense, and monitored by the U.S. Air Force Office of Scientific Research under Contract F44620-72-C-0019 with the University of Michigan's Human Performance Center. Requests for reprints should be sent to Robert G. Pachella, Human Performance Center, 330 Packard Road, Ann Arbor, Michigan 48104. lengthy. In this case, the subject sets up a serial buffer in short-term memory containing the most recent $S-R$ codes. A self-terminating serial search of this buffer is carried out, resulting in considerable savings in time. If the scan of the serial buffer is unsuccessful, the slower retrieval from long-term memory is carried out. It is the serial buffer that is sensitive to factors such as stimulus probability and number of alternatives. Thus, the Theios model makes differential predictions regarding the effect of variables like stimulus probability on tasks involving naming and manual responses to alphanumeric stimuli. Naming tasks are hypothesized to be independent of such variables, whereas tasks employing manual responses show systematic differences in response speed when factors affecting the informational content of the display are manipulated. It is the former task, the naming of alphanumeric stimuli, which is the concern of the present paper.

The Theios conceptualization of the difference in processing between naming and less compatible tasks such as button-pressing rests crucially on experimental evidence suggesting that reaction time (RT) for the naming task is independent of stimulus probability and number of stimulus alternatives. A review of the research in this area will reveal, however, that the hypothesized independence is still very much an empirical question.

Several studies (Brainard, Irby, Fitts, \& Alluisi, 1962; Morin \& Forrin, 1962; Mowbray, 1960; Theios, 1973) have shown naming RT to be independent of the number of stimuli. However, both Hellyer (1963) and Morin, Konick, Troxell, and McPherson (1965) found a small but significant increase in RT as the number of alternatives increased. Fitts and Switzer (1962) attempted to investigate under what conditions 
the independence of naming RT from the number of alternative stimuli was obtained. In a careful series of studies, they demonstrated an effect of subset familiarity on the RT/number of stimuli function. That is, when the sets of stimuli in the various conditions formed an unfamiliar grouping (e.g., the letters $E, B, P$ or the digits 2 and 7 , RT was independent of the size of the set. However, when the stimulus subsets were familiar (e.g., the letters A, B, $\mathrm{C}$ or the digits 1,2 ), RT did increase with the number of alternative stimuli. Fitts and Switzer argued that the subject, when given an unfamiliar subset, responds to the size of the total stimulus set rather than to the size of the actual stimulus subset. Thus, because of extensive prior experience with digits, the subset 2 and 7 seems as though it is drawn from the set 1 through 9, and the subject adopts a set appropriate to the 9 alternative case.

Results consistent with the Fitts and Switzer conceptualization are reported in a study by Forrin (1975). He found that when the stimuli were sets of digits, naming reaction time was the same for stimulus sets of four and eight alternatives. In contrast, naming RT was significantly increased when the eight-alternative set included stimuli from two different categories (letters and digits). Forrin discussed his results in terms of subset familiarity. $\mathrm{He}$ argued that the addition of stimuli from a different category increases the effective stimulus set size. The addition of items from the same category fails to achieve an effective stimulus set size increase because, regardless of the size of the subset, the subject develops an expectation for any member of the total stimulus ensemble. Thus, the subjective stimulus set size is equal in all conditions. The Forrin (1975) result is a compelling demonstration of the subset familiarity effect.

The above discussion takes on added relevance when it is noted that most of the studies indicating an independence of naming RT from the number of alternatives have employed unfamiliar subsets and are thus open to an explanation in terms of subset familiarity. Such an interpretation does not imply a difference in processing mechanisms associated with naming and less compatible tasks, as does the Theios model. It seems that the results of experiments that vary the number of alphanumeric stimuli to be named do not unambiguously support the notion of a time-invariant content-addressable name code to response code translation.

The situation with respect to the effect of stimulus probability on naming RT is even more questionable. Studies by Forrin, Kumler, and Morin (1966) and Theios $(1973,1975)$ have found the time to name digits to be independent of stimulus presentation probability. However, a significant effect of stimulus probability on naming RT was observed by Fitts,
Peterson, and Wolpe (1963) in a between-subjects design, and by Miller and Pachella (1973) in a within-subjects design. It would be fruitful to investigate whether the inconsistencies in the above results might be due to the presence of systematic artifacts in any of the experiments.

The most directly comparable of the conflicting studies are those of Miller and Pachella (1973) and Theios $(1973,1975)$. It should be noted first that the effect reported by Miller and Pachella was small (about $15 \mathrm{msec}$ ), although statistically significant. Thus, Theios is perhaps making a Type I error in accepting the invariance relation. Second, there are reasons why the Theios studies do not unequivocally substantiate the claim that naming $R T$ is independent of stimulus probability. The reported RTs from these experiments were fairly fast (approximately $350 \mathrm{msec}$ in the 1973 study and $385 \mathrm{msec}$ in the 1975 study), and the error rates are rather high for this type of task $(1 \%-6 \%$ in the 1973 study and $1 / 2 \%-21 / 2 \%$ in the 1975 study). More important, in both experiments the error rates were correlated with experimental conditions in the direction that larger error rates corresponded with low probabilities. Therefore, it is possible that an artifactual speed-accuracy trade-off was responsible for Theios' invariant RTs (Pachella, 1974).

The present study attempts to investigate whether errors are systematically related to stimulus probability levels in a naming task. This was accomplished by the use of a response-deadline procedure that has been used to control the speed of responses across experimental conditions (Pachella \& Fisher, 1972). The procedure makes it possible to look at the effect of stimulus probability on error rates when reaction time is held relatively constant.

\section{METHOD}

\section{Subjects}

The subjects were 20 paid volunteers recruited on the University of Michigan campus.

\begin{abstract}
Apparatus
A PDP-1 computer controlled the presentation of stimuli while recording responses and response latencies. The stimuli were the digits $1-8$ presented on a Hewlett-Packard 1311A display device. The digits were approximately $1 \mathrm{~cm}$ high. Subjects sat about $65 \mathrm{~cm}$ from the display device. A voice key attached to the computer measured the vocal RTs. In the accuracy condition, the simulus left the screen after the subjects response, and approximately $2 \mathrm{sec}$ later another stimulus appeared. In the speed emphasis condition, after the subject responded he was given the feedback "fast" or "slow" in the lower left corner of the display device depending on whether he had beaten a 375-msec deadline. The experimenter recorded the accuracy of the subject's responses on a microswitch keyboard attached to the computer. The experimenter was seated directly behind the subject.
\end{abstract}

\section{Procedure}

Twelve subjects served in the accuracy condition and eight 
subjects served in the speed condition of the experiment. In the accuracy condition, subjects were told to respond to the digit as soon as possible after it appeared on the screen, while trying not to make any errors. In the speed emphasis condition, subjects were told to make sure to beat the deadline with their responses.

Each subject was run in four blocks of 200 trials on the same day. The stimulus probabilities used in each block were $.275, .125, .075$, .025 . Two digits occurred at each level of stimulus probability. These probabilities were held constant for all four blocks for a given subject. The stimulus probabilities assigned to specific digits were counterbalanced across subjects. Stimulus sequences were generated by a pseudorandom computer algorithm, subject only to the constraint that the frequency of each stimulus in each 200-trial block correspond to its assigned frequency. Between blocks, subjects were given 3-min rest periods.

\section{RESULTS}

For each block of trials, mean RT and percent error were computed at each level of stimulus probability. These values, averaged across subjects in both the accuracy and speed emphasis conditions, are plotted in Figure 1. An analysis of variance on the RTs of the accuracy condition showed main effects of Stimulus Probability $[F(3,33)=4.02, p<.025]$ and Practice $[F(3,33)=3.47, p<.05]$. The Stimulus Probability by Practice interaction was not significant. An analysis of variance on the RTs of the speed emphasis condition showed a significant main effect of Practice $[F(3,21)=12.01, p<.001]$. The effect of Stimulus Probability $[F(3,21)=2.77]$ did not reach statistical significance, although the RTs increased monotonically with decreasing probability. The Practice by Stimulus Probability interaction was not significant. An analysis of variance on the error rates of the speed emphasis condition showed the effect of Stimulus Probability to be significant $[F(3,21)=7.88$, $\mathrm{p}<.01]$. Error rates increased monotonically with decreasing stimulus probability. Neither the effect of Practice nor the Practice by Stimulus Probability interaction was significant.

\section{DISCUSSION}

The results of the present experiment clearly indicate that a stimulus probability effect can be manifested in a naming task either by increased RTs or as increased error rates with low-probability stimuli. In the accuracy condition a significant effect of stimulus probability on RT was observed. The magnitude of the effect was similar to that reported by Miller and Pachella (1973). The response-deadline technique of the speed emphasis condition was not entirely successful in equating RTs across probability levels. Although the effect of stimulus probability was not significant in this condition, RT did increase systematically with decreasing stimulus probability. The failure of the responsedeadline technique to achieve complete equality of RTs across probability levels can only be interpreted as further evidence that the time to name alphanumeric stimuli is not independent of the probabilities of the stimuli. This conclusion is strongly supported by the results of the analysis of variance on the error rates. They were monotonically related to the probability level and the differences in error rates were highly significant. This result indicates how stimulus probability affects a dependent variable that covaries with RT and, in addition, demonstrates the manner in which

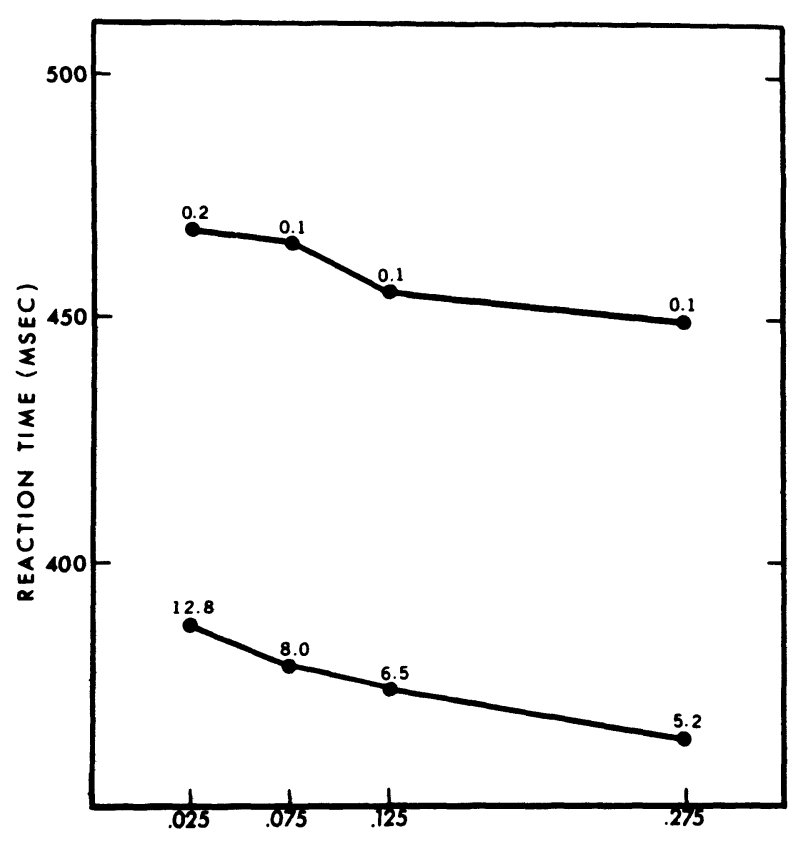

STIMULUS PROBABILITY

Figure 1. Reaction time as a function of stimulus probability. The upper curve is the accuracy condition, the lower curve in the speed condition. Numbers above each point indicate the percentage of errors for each condition.

speed-accuracy considerations may have been operating in the Theios $(1973,1975)$ experiments.

A resolution of at least some of the ambiguities in previous experiments manipulating stimulus probability in naming tasks is suggested by the present results. Investigators must look for effects of stimulus probability on both the RTs and the error rates generated in the experiment. The present study demonstrated probability effects on both of these covarying dependent variables. It appears as if stimulus probability does systematically affect the time to name alphanumeric stimuli. In addition, other research points to the conclusion that number of stimulus alternatives also affects naming RT. The experiments showing RT to be independent of the number of stimulus alternatives are subject to the criticism that the manipulation did not increase the subjective ensemble size due to the use of unfamiliar subsets. Experiments that are not subject to this criticism have shown a significant number of alternatives effect (Fitts \& Switzer, 1962; Forrin, 1975). In summary, it must be concluded that the time to name alphanumeric stimuli is affected by variables associated with the informational content of the display.

The above conclusion presents problems for the Theios (1975) model of information processing in speeded tasks. It will be recalled that this model defines two stages that are involved in encoding a stimulus. The first is an input stage, in which the physical stimulus is transformed by the nervous system to a stimulus code that can be used in memory. The second is an identification stage, in which the stimulus code is transformed into the name code of the stimulus. These two encoding stages are assumed to be affected by stimulus variables like clarity and intensity, but are independent of stimulus probability and number of alternatives. In naming tasks the name code to response code translation takes place via a content addressable retrieval process that also is independent of probability and number of alternatives. Since all the subprocesses involved in the naming task are independent of informational variables, the total time to respond verbally to an alphanumeric stimulus must be independent of stimulus probability. The result reported in the 
present experiment strongly suggests a modification of the Theios model in this respect. An elaboration of either the identification or the translation stages seems necessary.

Additional results from the Miller and Pachella (1973) experiment suggest that at least part of the stimulus probability effect manifested in naming tasks is a result of the operation of the identification stage. They found that, when the stimuli were degraded, the effect of stimulus probability on naming RT was much greater. This interaction, interpreted in terms of the additive-factor methodology introduced by Sternberg (1969), would indicate that stimulus probability and stimulus degradation affect a stage in common. Since the identification stage of the Theios model is affected by stimulus quality, the Miller and Pachella result suggests that this stage is the locus of at least part of the stimulus probability effect.

\section{REFERENCES}

Brainard, R. W., Irby, T. S., Fitts, P. M., \& Alluisi, E. A. Some variables influencing the rate of gain of information. Journal of Experimental Psychology, 1962, 63, 105-110.

Fitts, P. M., Peterson, J. R., \& Wolpe, G. Cognitive aspects of information processing: II. Adjustments to stimulus redundancy. Journal of Experimental Psychology, .1963, 65, 507-514.

Fitts, P. M., \& SwItzer, G. Cognitive aspects of information processing: $I$. The familiarity of $S-R$ sets and subsets. Journal of Experimental Psychology, 1962, 63, 321-329.

ForRIN, B. Naming latencies to mixed sequences of letters and digits. In P. Rabbitt \& S. Dornic (Eds.), Attention and performance V. New York: Academic Press, 1975.

Forrin, B., Kumler, M., \& Morin, R. E. The effects of response code and signal probability in a numeral-naming task. Canadian Journal of Psychology, 1966, 20, 115-124.
Hellyer, S. Stimulus-response coding and amount of information as determinants of reaction time. Journal of Experimental Psychology, 1963, 65, 521-522.

Miller, J. O., \& Pachella, R. G. Locus of the stimulus probability effect. Journal of Experimental Psychology, 1973, 101, 227-231.

Morin, R. E., \& Forrin, B. Mixing of two types of S-R associations in a choice reaction time task. Journal of Experimental Psychology, 1962, 64, 137-141.

Morin, R. E., Konick, A., Troxell, N., \& McPherson, S. Information and reaction time for "naming" responses. Journal of Experimental Psychology, 1965, 70, 309-314.

Mowbray, G. H. Choice reaction times for skilled responses. Quarterly Journal of Experimental Psychology, 1960, 12, 193-202.

Pachella, R. G. The interpretation of reaction time in information processing research. In B. Kantowitz (Ed.), Human information processing: Tutorials in performance and cognition. Hillsdale, N.J: Lawrence Erlbaum Associates, 1974.

Pachella, R. G., \& Fisher, D. Hick's law and the speedaccuracy trade-off in absolute judgment. Journal of Experimental Psychology, 1972, 92, 378-384.

STERNBERG, S. The discovery of processing stages: Extensions of Donder's method. In W. G. Koster (Ed.), Attention and performance II. Amsterdam: North Holland, 1969.

Theros, J. Reaction time measurements in the study of memory processes: Theory and data. In G. Bower (Ed.), The psychology of learning and motivation: Advances in research and theory (Vol. 7). New York: Academic Press, 1973.

Theros, J. The components of response latency in simple human information processing tasks. In P. Rabbitt \& S. Dornic (Eds.), Attention and performance $V$. New York: Academic Press, 1975.

(Received for publication June 5, 1976.) 